\title{
PRIMER REGISTRO FÓSIL DE PLIOMETANASTES SP. (MAMMALIA, XENARTHRA, MEGALONYCHIDAE) PARA EL MIOCENO SUPERIOR DE COSTA RICA, AMÉRICA CENTRAL. UNA NUEVA PISTA EN LA COMPRENSIÓN DEL PRE-GABI
}

\author{
FIRST FOSSIL RECORD OF PLIOMETANASTES SP. (MAMMALIA, XENARTHRA, \\ MEGALONYCHIDAE) FROM THE UPPER MIOCENE OF COSTA RICA, \\ CENTRAL AMERICA. A NEW CLUE TO UNDERSTAND THE PRE-GABI
}

\author{
Cesar A. Laurito ${ }^{1,2^{*}}$ \& Ana L. Valerio ${ }^{2}$ \\ ${ }^{1}$ Instituto Nacional de Aprendizaje \\ ${ }^{2}$ Departamento de Historia Natural, Museo Nacional de Costa Rica. \\ Apdo. 749-1000, San José \\ *Autor para contacto: cesarlaurito@ice.co.cr
}

(Recibido: 21/03/2012 ; aceptado: 17/12/2012)

\begin{abstract}
Two lower molar teeth and a unique postcranial remain of Pliometanastes cf. P. protistus Hirschfeld \& Webb, 1968, are described from the San Gerardo de Limoncito Locality (Early Hemphillian) in the Curré Formation near of the town of San Vito, Southern Costa Rica.

This found changes our vision about the route of dispersion of the earliest South American mammals that reached North America and confirms the Costa Rican-Panama Island Arc like the most effective path of migration in spite of the relatively broad ocean gaps.

Keywords: Mammalia, Xenarthra, Megalonychidae, Pliometanastes, Upper Miocene, Costa Rica, GABI.

RESUMEN: Se describen molares inferiores y un único resto post-craneal de Pliometanastes cf. P. protistus Hirschfeld \& Webb, 1968, procedentes de la localidad de San Gerardo de Limoncito (Hemphilliano temprano), cerca de la ciudad de San Vito en el sur de Costa Rica.

Este hallazgo, cambia nuestro entendimiento de la ruta de dispersión de los primeros mamíferos suramericanos que alcanzaron América del Norte y validan al Arco de Islas Costa Rica-Panamá, como la vía más idónea de migración a pesar de las relativamente amplias barreras oceánicas.

Palabras clave: Mammalia, Xenarthra, Megalonychidae, Pliometanastes, Mioceno Superior, Costa Rica, GABI.
\end{abstract}




\section{INTRODUCCIÓN}

Tres géneros de perezosos terrestres, Megalonyx y Pliometanastes de la familia Megalonychidae y el Mylodontidae Thinobadistes fueron descritos como los primeros inmigrantes suramericanos en América del Norte (Hirschfeld, 1981). Ellos arribaron al menos 6.0 Ma antes del establecimiento del Istmo de Panamá, durante el Hemphilliano temprano, y 3.0 Ma previo al inicio del Gran Intercambio Faunístico entre las Américas (Marshal et al., 1979) o Pre-GABI por sus siglas en inglés. Estos xenarthras también son considerados como los Heraldos del intercambio faunístico sensu Webb (1976), por su migración temprana y su registro fósil relativamente antiguo en la península de Florida, en California y otros lugares del subcontinente norteamericano; desde entonces se ha especulado sobre la posible vía de dispersión de estos perezosos terrestres, considerándose las Antillas Mayores y el Levantamiento de Aves como la ruta migratoria más probable (MacPhee \& Iturralde-Vinnent, 1994, 1995; MacPhee, 2005).
En el último decenio, el Museo Nacional de Costa Rica ha realizado trabajos de campo en la localidad faunística de San Gerardo de Limoncito, cantón de Coto Brus, provincia de Puntarenas, en las coordenadas geográficas 851'19.6”N/8304'51.9'W (Fig. 1); recuperándose una importante colección paleontológica de edad Hemphilliano temprano con mamíferos tanto de abolengo norteamericano como sudamericano (Laurito \& Valerio, 2005, 2008, 2010; Valerio \& Laurito, 2008; Valerio, 2010).

Entre los elementos de abolengo suramericano se han recuperado restos de Pampatheriidae, Megatheriidae, Megalonychidae y probable Mylodontidae, todos de edad Pre-GABI. Entre los restos de Megalonychidae, destacan dos molares inferiores izquierdos, el CFM-2017 correspondiente a un $1 \mathrm{M}$ y el CFM-1000 correspondiente a un $3 \mathrm{M}$; un astrágalo izquierdo con código CFM-2645, todos depositados en la Colección de Fósiles de la Sección de Geología, Departamento de Historia Natural del Museo Nacional de Costa Rica y analizados en el presente trabajo.
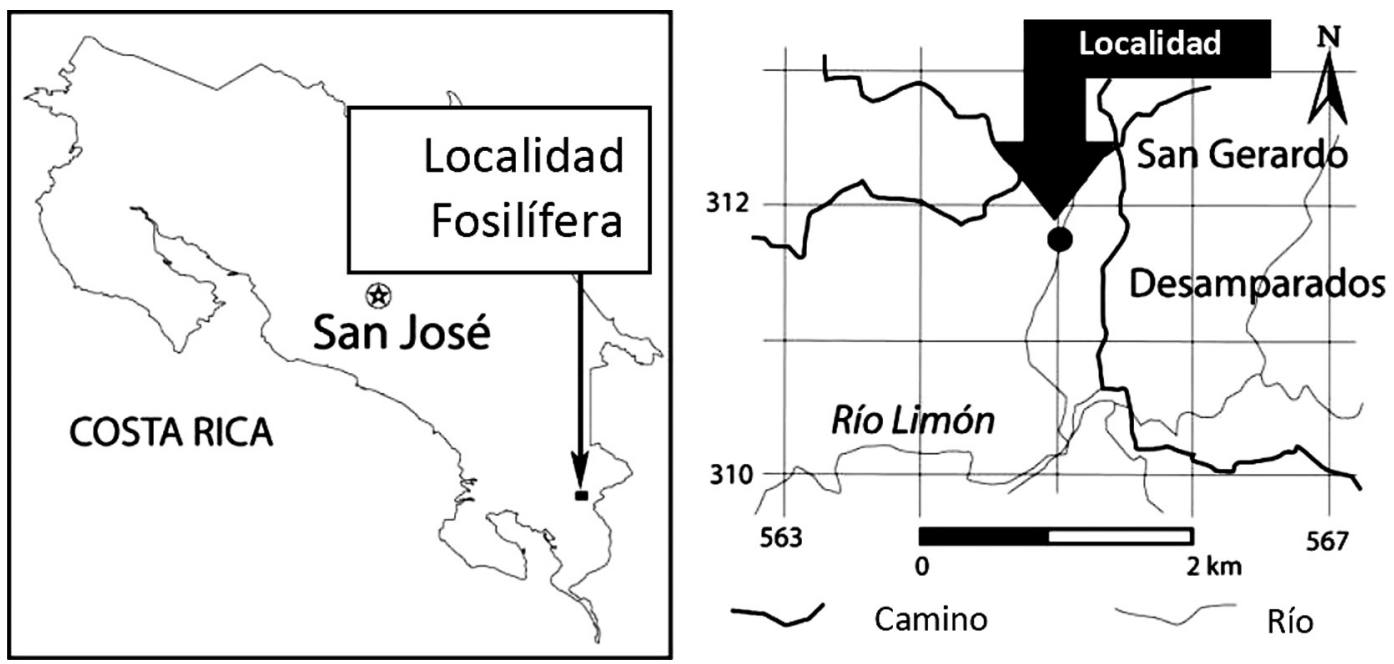

Fig. 1: Mapa de ubicación de la localidad fosilífera de San Gerardo de Limoncito, cantón de Coto Brus, provincia de Puntarenas. 


\section{PALEONTOLOGÍA}

Clase MAMMALIA Linnaeus, 1758

Orden XENARTHRA Cope, 1889

Suborden TARDIGRADA Latham y Davies en Forster, 1795

Infraorden PILOSA Flower, 1883

Familia MEGALONYCHIDAE Zittel 1892

Subfamilia MEGALONYCHINAE Trouessart 1904

Pliometanastes Hirschfeld \& Webb 1968

Pliometanastes cf. P. protistus Hirschfeld \& Webb 1968

Especie tipo: Pliometanastes protistus Hirschfeld \& Webb 1968

Localidad tipo: McGehee Farm, Alachua County, Florida

\section{Descripción}

Molares inferiores izquierdos: el CFM-2917 (Figs. 2a-a"') corresponde a un ${ }_{1} \mathrm{M}$ roto (Cuadro 1), pseudo-cilíndrico con la superficie oclusal bien preservada y de contorno subrectangular, pentagonal alargada a grosso modo la cara anterior es bastante plana y más angosta que la cara comisural que es amplia y redondeada. El CFM-1000 (Figs. 2b-b"') corresponde a un 3M roto, de contorno subrectangular, con la cara vestibular bastante plana y la lingual redondeada y ligeramente angulosa; la cara anterior o mesial es redondeada y abierta, y la cara comisural es bastante plana y presenta una muesca que define el primer tercio del lado vestibular de la superficie oclusal.

Astrágalo izquierdo: CFM-2645, completo y bien conservado con la cara medial más corta que la lateral y con una depresión somera entre las facetas tibial y navicular. La faceta interna del calcáneo es ligeramente cóncava y un poco con forma de diamante, con el ápice curvado hacia el aspecto externo del calcáneo y está conectada a la faceta externa por una loma. La faceta navicular es oval con forma similar a una lágrima (Figs. 3a-e).

\section{DISCUSIÓN}

De acuerdo con McDonald \& Naples (2007) una identificación positiva del género Pliometanastes requiere de la preservación del diente caniniforme, el cual no hemos hallado. Sin embargo, si existen sutiles diferencias entre los primeros molares inferiores de Megalonyx y Pliometanastes; de acuerdo a Hirschfeld (1981), los $1 \mathrm{M} 1$ son sub-rectangulares angosto anteriormente y ancho posteriormente, en tanto los $3 \mathrm{M} 3$ no se diferencian de los terceros molares inferiores de Megalonyx curvidens.

Al comparar el $1 \mathrm{M}$ con los 1M1 de Megalonyx curvidens, la especie contemporánea de Megalonychidae de América del Norte, las diferencias más notables son el contorno del molar y de su superficie oclusal que son notablemente redondeadas casi elípticas en $M$. curvidens, en tanto que en Pliometanastes protistus Hirschfeld $\&$ Webb, 1968 el contorno es groseramente trapezoidal o sub-rectangular.

El contorno del 3M difiere muy poco del contorno de los 3M3 de Megalonyx curvidens, siendo en esta última más redondeado y cuadrangular. También se observa en la cara comisural del 3M de Pliometanastes protistus, una muesca o inflexión en su tercio vestibular que no se encuentra en Megalonyx curvidens.

Por otra parte, la forma del astrágalo recuperado es indiferenciable del astrágalo descrito e ilustrado para Pliometanastes protistus por Hirschfeld \& Webb (1968), pero sus dimensiones son ligeramente más grandes; según estos autores, este tipo de astrágalo difiere del astrágalo del género Megalonyx por ser más redondeado, con 

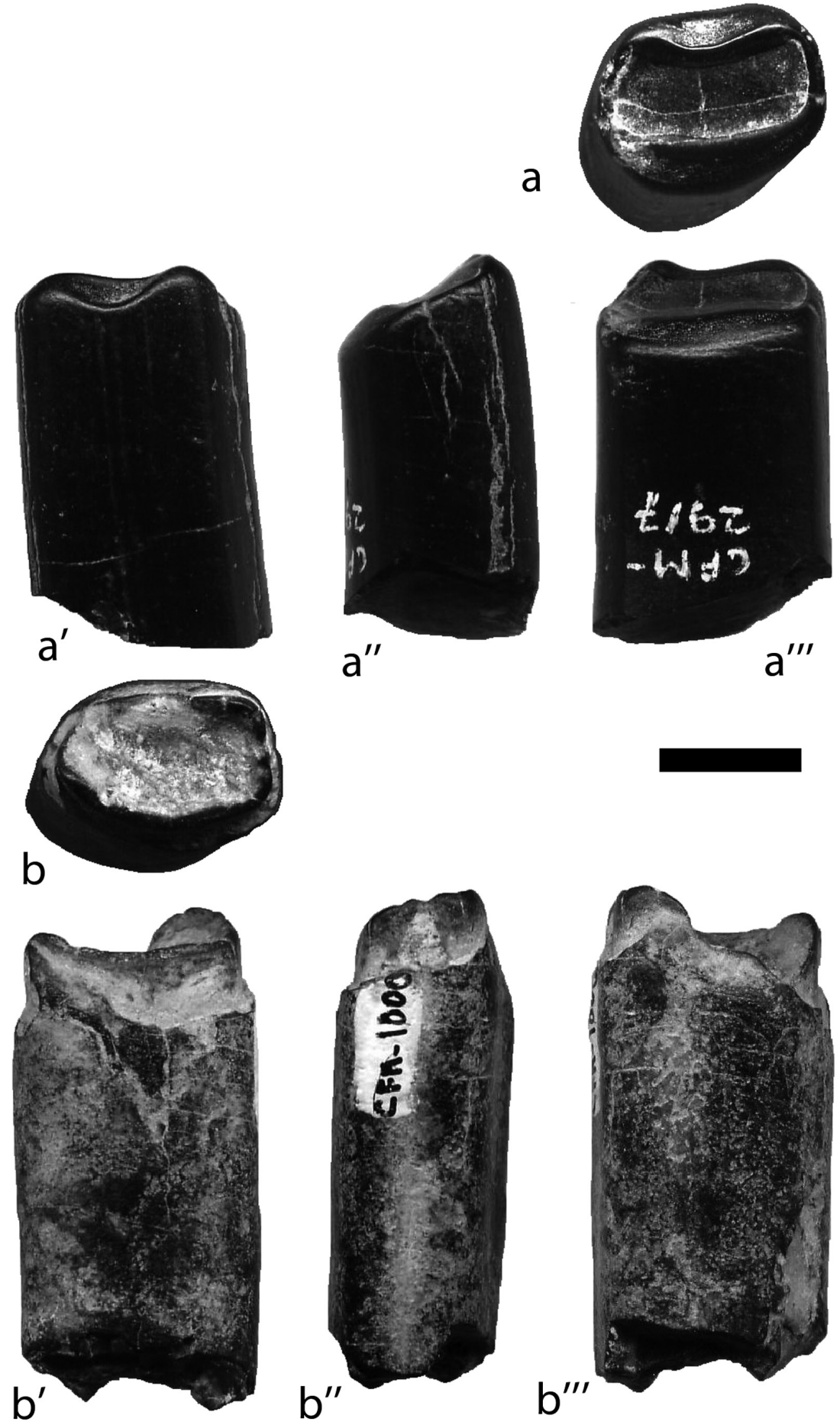

Fig. 2: CFM-2917 molar ${ }_{1}$ M en vista, a: oclusal, a': mesial, a": lingual y a"': comisural; CFM-1000 molar ${ }_{3} \mathrm{M}$ en vista, b: oclusal, b': mesial, b": labial y b"': comisural. Escala visual $1 \mathrm{~cm}$. 
Cuadro 1

Medidas de los molares y astrágalo de Pliometanastes $\mathrm{cf}$. P. protistus

\begin{tabular}{lc}
\hline Molar/Astrágalo & Dimensiones (mm) \\
\hline Molar ${ }_{1} \mathrm{M}(\mathrm{CFM}-2917)$ & 16,88 \\
Longitud vestíbulo lingual & 13,27 \\
Longitud antero posterior & 17,62 \\
\hline Molar ${ }_{3} \mathrm{M}$ (CFM-1000) & 12,37 \\
Longitud vestíbulo lingual & 61,82 \\
Longitud antero posterior & 40,75 \\
\hline Astrágalo izquierdo (CFM-2645) & 28,23 \\
Máximo diámetro antero posterior paralelo a la faceta fibular & 28,72 \\
Máximo diámetro transverso medido en ángulo recto a la faceta fibular & 37,83 \\
Profundidad de la faceta fibular, extremo distal & \\
Máximo diámetro transverso de la cabeza & \\
Longitud antero posterior de la faceta calcaneal externa &
\end{tabular}

el lado medial más corto que el lateral (Hirschfeld \& Webb, 1968, p. 277), siendo uno de los pocos huesos de post-cráneo que permiten algún tipo de diferenciación entre los géneros Pliometanastes y Megalonyx.

\section{Distribución paleogeográfica y estratigráfica}

Pliometanastes ha sido registrado junto con Thinobadistes (Mylodontidae), en el Hemphilliano temprano Hh1 (8.0-9.0 Ma) de la localidad fosilífera de McGehee Farm en la península de Florida sensu Hirschfeld \& Webb (1968) y Morgan (2005), también se han registrado en otras localidades de Estados Unidos, pe. Pliometanastes se registra en el Hemphilliano temprano de California de acuerdo con Hirschfeld (1981); Hirschfeld \& Webb (1968); Webb (2006) y Thinobadistes en Texas (Webb, 1989) con edades similares. En México, Carranza-Castañeda \& Miller (2004) registran el hallazgo de Pliometanastes en el estado de Nuevo León con una edad de alrededor de 8.0 Ma y Carranza-Castañeda et al. (2008), registran la asociación de Pliometanastes sp. junto con Dinohippus mexicanus y Calippus hondurensis en la localidad de Juchipila en el estado de Zacatecas (Fig. 4).

\section{Revisión de la edad de la localidad de San Gerardo de Limoncito}

La primera aparición del género Pliometanastes en América del Norte define el inicio del Hemphilliano temprano Hh1, junto con el registro del Mylodontidae Thinobadistes sensu Tedford et al., (1987); estos dos géneros son considerados coetáneos (Marshal et al., 1979; Webb, 1985). Hirscheld (1981) reporta que Pliometanastes ocurre estratigráficamente, $4 \mathrm{~m}$ por debajo de una toba datada radiométricamente por K-Ar en $8.19+0.16$ Ma de la Formación Merthen en California. La última aparición de Pliometanastes en el registro fósil data de $6.1 \mathrm{Ma}$ de la localidad Box T Ranch, Lipscomb County, Texas de acuerdo con McDonald \& Naples (2007). El registro más austral hasta ahora era el de Juchipila, México (Carranza-Castañeda et al., 2006; 2008), quienes asignan a esa localidad una edad Hemphilliano temprano (Hh1-2).

La edad mínima de la localidad de San Gerardo de Limoncito, fue establecida por Valerio (2010) y Laurito \& Valerio (2010) como Hemphilliano temprano tardío por la asociación de los caballos Protohippus gidleyi Hulbert, 1988 [Cl2-Hh2 equivalente a 9.48-6.57 Ma (Maguire, 2008; MacFadden \& Dobie, 1998)], Calippus 

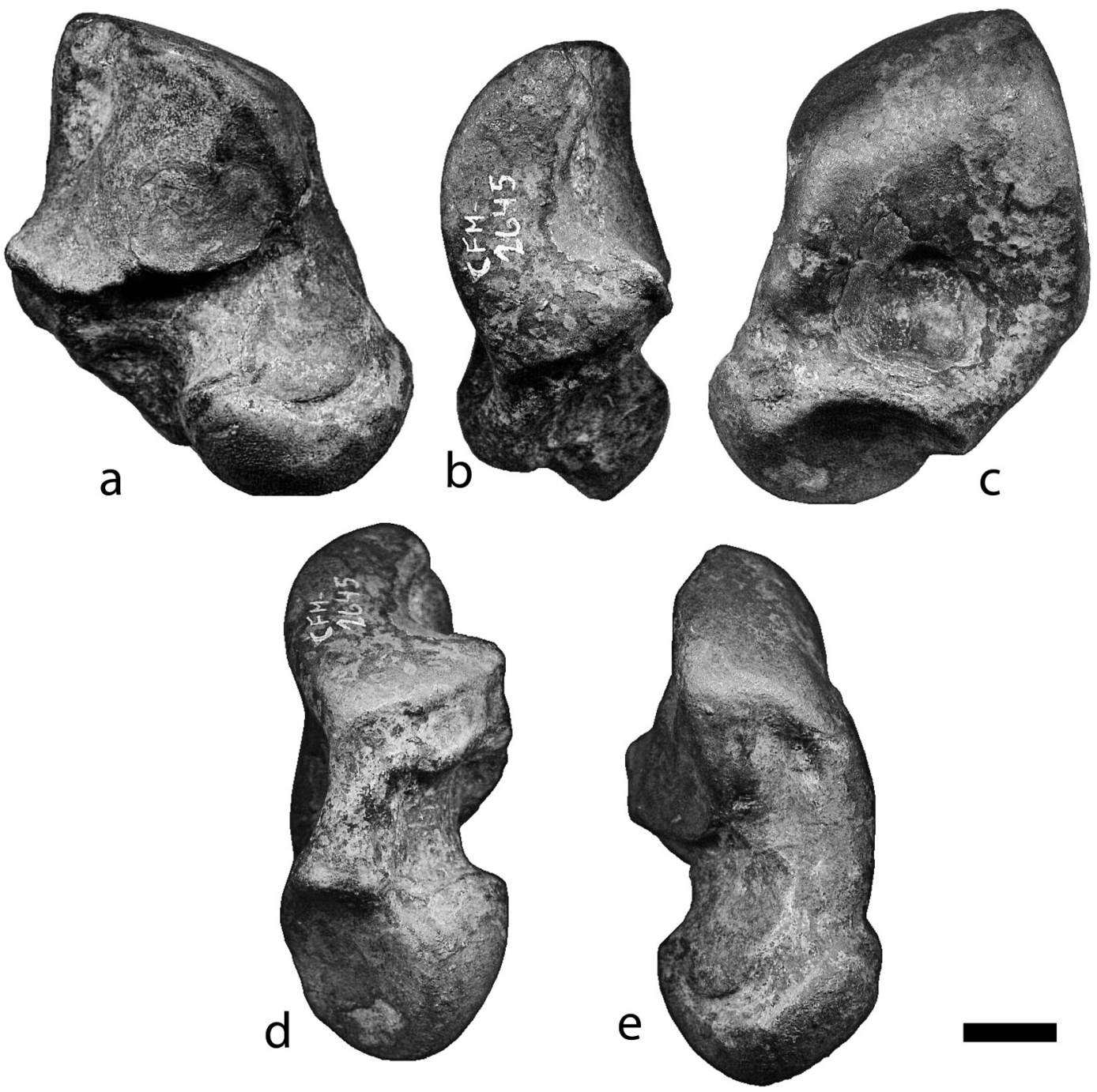

Fig. 3: Astrágalo izquierdo de Pliometanastes cf. P. protistus Hirschfeld \& Webb 1968, CFM- 2645, en vista a. calcaneal; b. fibular; c. tibial; d. lateral y e. medial. Escala visual $1 \mathrm{~cm}$.

hondurensis (Olson \& McGrew, 1941) [rango estratigráfico $\mathrm{Cl} 2-\mathrm{Hh} 2$ sugerido por Maguire (2008) equivalente a 9.48-6.57 Ma (cf. Alroy, 2000)] y Dinohippus mexicanus (Lance, 1950) [Hh3-Hh4,
Hemphilliano tardío por Johnston \& Savage (1955), May \& Repenning (1982), Dalquest \& Mooser (1980), Lindsay et al. (1984) y Lindsay (1984); equivalente a 6.57-4.8 Ma]. 


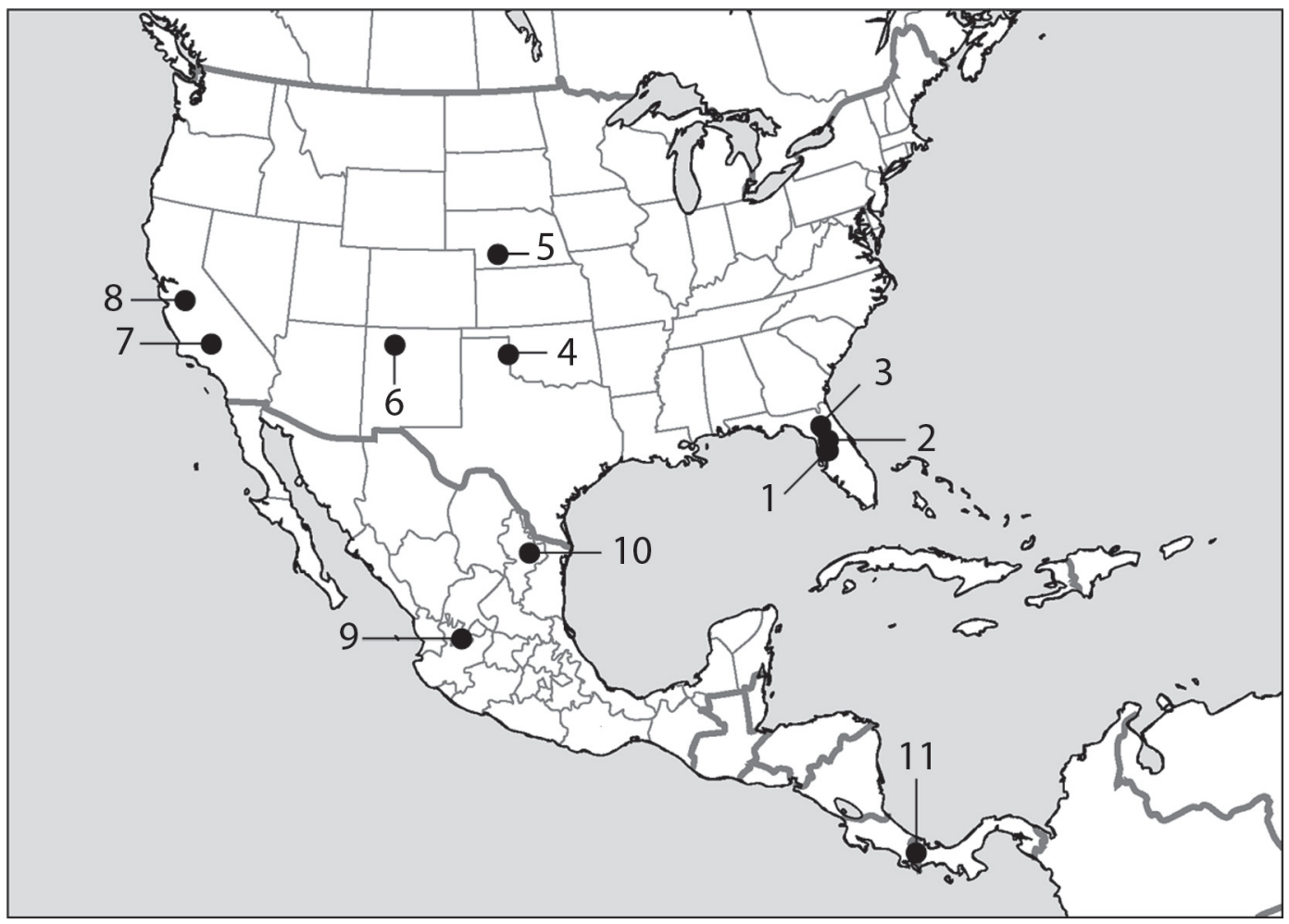

Fig. 4: Mapa de distribución paleobiogeográfico del género Pliometanastes y sus especies, P. protistus: 1. Marion County, Florida; 2. Moss Acres Racetrack, Alachua Formation y Dunnellon Phosphate Mining District, ambas en Marion County, Florida; 3. McGehee Farm (Hirschfeld \& Webb, 1968) y Alachua County (localidad de Haile 5A, 19A), Florida (MacFadden, 1982); 4. Lipscomb County, Texas (Schultz, 1990); 5. Pliometanastes sp. en Frontier County, Nebraska; 6. Pliometanastes sp. en Black Mountain (Tedford, 1981, Tedford \& Barghoorn, 1993; Wang et al., 1999) y Pliometanastes galushai en Rio Arriba County (MacFadden, 1977), ambas localidades en Chamita Formation, New Mexico; 7. Pliometanastes sp. en Upper Caliente Formation, Kern County, California (Hirschfeld \& Webb, 1968); 8. P. protistus en Mehrten Formation, Stanislaus County, California (Hirschfeld \& Webb, 1968); 9. Posible Pliometanastes sp. en la Formación Juchipila, Estado de Zacatecas, México (Carranza-Castañeda et al., 2008); 10. Pliometanastes sp. en noreste del estado de Nuevo León (Carranza-Castañeda \& Miller, 2004); y 11. Pliometanastes cf. protistus en la localidad fosilífera de San Gerardo de Limoncito, cantón de Coto Brus, en la Formación Curré, Costa Rica (presente trabajo).

Fundamentado en lo anterior, a la localidad de San Gerardo de Limoncito se le asigna una edad biocronológica Hemphilliano temprano, cuya edad máxima sería el Hemphilliano temprano-temprano (Hh1) y una edad mínima Hemphilliano temprano-tardío $(\mathrm{Hh} 2)$ equivalente a un rango de edad de entre 8.5 a 6.5 Ma.

\section{Discusión sobre la ruta de dispersión y el arribo temprano de Pliometanastes a Norte América}

Normalmente se aceptaba que la migración de los xenarthras Pliometanastes y Thinobadistes a la Florida siguió una ruta de dispersión a través de 
las Antillas Menores y Mayores (Webb, 1985), ello parece validarse por el notable registro de xenarthras fósiles en las Antillas. De hecho, los registros más antiguos de xenarthras en las Antillas Mayores datan del Oligoceno Temprano de Puerto Rico y Mioceno Temprano de Cuba con alrededor de 17.5-18.5 Ma (cf. MacPhee \& Iturralde-Vinnent, 1994; White \& MacPhee, 2001; MacPhee et al., 2003) y se acepta que arribaron durante el Oligoceno, cuando el nivel del mar era extraordinariamente bajo y la configuración paleogeográfica de las Antillas Mayores permitió la conexión de estas con el levantamiento de Aves (Aves Ridge) y el norte de Sudamérica (MacPhee \& Iturralde-Vinnent, 1994, 1995; MacPhee, 2005), territorio al que dichos autores denominan Gaarlandia. Pero, no hay evidencia faunística de que las Antillas en general hayan estado alguna vez estrictamente conectadas con las áreas continentales adyacentes en el Cenozoico, mediante un territorio emergido continuo (Patterson \& Pascual, 1968) y es claro que la colonización de estas islas se dio por migración accidental (Simpson, 1956) de organismos saltadores de islas (Pascual, 2006). Recientemente, MacPhee \& Iturralde-Vinnent (2012) con base en el análisis de los relojes moleculares de diversos vertebrados terrestres, asumen que una breve conexión de Gaarlandia con América del Sur se dio entre 35 y $33 \mathrm{Ma}$, pues es notable la invasión de vertebrados terrestres en el límite Eoceno/Oligoceno, lo que no pudieron determinar es si esa invasión fue vía marítima o terrestre.

Por otra parte, este hecho confirma que los perezosos se dispersaron a través de amplios pasos marinos que actuaron como barreras acuáticas y como la misma Hirschfeld (1981) concluyó, la dispersión por el Caribe de estos xenarthras los ubica como saltadores de islas, ya sea como fuertes nadadores o por medio de balsas naturales (= funerales vikingos). Sin embargo, Hirschfeld (1981) concluye que existen diferencias taxonómicas entre las formas de las Antillas y las de América del Norte, de allí que la ruta de dispersión de las formas norteamericanas fue probablemente vía América Central (CarranzaCastañeda \& Miller, 2004).
El hallazgo costarricense de Pliometanastes, valida estas últimas suposiciones, confirmando que los Megalonychidae tempranos arribaron a América Central meridional en el Hemphilliano temprano, al final de la época de Colisión temprana o Syn-colisión (Mioceno Medio temprano hasta el Mioceno Tardío, $<14.8 \mathrm{Ma}$ a 7.1 Ma) del Arco de Islas Costa Rica - Panamá con el NW de América del Sur, según los modelos de los escenarios tectónicos planteados por Coates et al. (2004). Además, se confirma que el sur del istmo de Panamá conformaba en esa época un archipiélago y una barrera oceánica que aunque importante fue superada por estos megalonychidos, ya sea como fuertes nadadores o saltadores de islas accidentales; hecho que ya habían logrado otros Megalonychidae al alcanzar las Antillas Mayores en el Oligoceno Temprano (MacPhee \& IturraldeVinnent, 1995). Además, es claro que no hay evidencia de arribo de xenarthras en América Central de previo al Hemphilliano temprano tardío (Laurito \& Valerio, 2012) y las localidades faunísticas más meridionales y antiguas del Istmo como las de la Formación Cucaracha en Panamá no muestran evidencia de elementos de abolengo suramericano a pesar de su cercanía paleogeográfica con dicho continente (MacFadden et al., 2012).

\section{¿Dónde se diferenció el género Pliometanastes? y ¿cuál fue el ancestro suramericano que dio origen al género Pliometanastes?}

Pliometanastes se caracteriza por una amplia distribución en el Hemphilliano temprano de América del Norte y se acepta que dio origen al género Megalonyx (McDonald, 2005; McDonald $\&$ Naples, 2007), a pesar de que Megalonyx había sido considerado como un inmigrante temprano de América del Sur (Hirschfeld, 1981), ahora se acepta que es endémico de América del Norte (Morgan, 2005), su registro más antiguo proviene de la fauna de Lemoyne en Nebraska y data de 6.7 Ma (Leite, 1990). El análisis cladístico de Gaudin (2004), sugiere que Megalonyx es el grupo hermano ("sister group") de Pliometanastes y éste el grupo hermano de Pliomorphus, que se 
supone es el género basal del grupo corona de todos los Megalonychidae de las Indias Orientales (sensu Gaudin, 2004), además confirma al género Pliomorphus como ancestral con respecto a Pliometanastes, todo ello es acorde con lo concluido por Hirschfeld \& Webb (1968).

De momento se asume que Pliometanastes migró de América del Sur, sin embargo este género no ha sido registrado en ese subcontinente y una nueva posibilidad es que este se haya diferenciado tempranamente en las islas del archipiélago que conformaban el sur de América Central, a partir de un Megalonychidae ancestral durante el Hemphilliano temprano.

\section{CONCLUSIONES}

El Megalonychidae Pliometanastes se describe por primera vez en América Central, este hallazgo representa el registro más austral del género en América del Norte y amplía el rango de edad de la fauna de la localidad de San Gerardo de Limoncito correspondiente al tope de la Formación Curré.

Este registro valida la suposición previa de que los Megalonychidae y en especial Pliometanastes, ingresaron vía el Istmo de Panamá casi $6.0 \mathrm{Ma}$ antes del cierre efectivo del mismo y la instauración del corredor biológico interamericano. Pero no verifica el sitio de evolución y aparición del género Pliometanastes, quedando la posibilidad de que el mismo se haya diferenciado en el sur de América Central a partir de un Megalonychidae ancestral proveniente de América del Sur.

El modo de dispersión es muy probable que se diera por el arribo accidental de estos perezosos terrestres, a la porción más meridional del archipiélago del Arco de Islas Costa Rica-Panamá, durante el Mioceno Superior.

El arribo de este y otros Xenarthras al sur de América Central, como es el caso de los Pampatheriidae, Mylodontidae y Megatheriidae, confirma una vez más la gran capacidad de estos mamíferos terrestres de superar grandes pasos marinos, ya sea como fuertes nadadores o afortunados sobrevivientes de balsas o funerales vikingos, que rápidamente se adaptaron a las condiciones climáticas de estas jóvenes tierras donde originaron especies nuevas y mejor adaptadas.

\section{AGRADECIMIENTOS}

Al Dr. Greg McDonald del National Park Service at the Hagerman Fossil Beds, Idaho, U.S. Department of the Interior, quién nos suministró importante material comparativo y bibliográfico y al Dr. Gabriel González Barba, Universidad Autónoma de Baja California Sur, México, por la revisión del texto y sus acertadas observaciones.

\section{REFERENCIAS BIBLIOGRÁFICAS}

ALROY, J., 2000: New methods for quantifying macroevolutionary patterns and processes.- Paleobiol. 26(4): 707-733.

CARRANZA-CASTAÑEDA, O.\& MILLER, W., 2004: Late Tertiary Terrestrial Mammals from Central Mexico and their relationship to South American Inmigrants.- Rev. Brasileira Paleont. 7(2): 249-261.

CARRANZA-CASTAÑEDA, O., ARANDA, J., AGUIRRE, G., \& NIETO, J., 2006: Estratigrafía del Terciario Tardío en la cuenca de Juchipila-Jalpa, Estado de Zacatecas y sus implicaciones para el Gran Intercambio de Faunas entre las Américas.Geos, 26(1): 132.

CARRANZA-CASTAÑEDA, O., WANG, X., ARANDA, J.J., TZENG, J., TRONCOSO, H. \& CERVANTES, J., 2008: Mastofauna del Terciario Tardío de la Formación Juchipila, Estado de Zacatecas y su correlación bioestratigráfica con las faunas del centro de México.- Geos, 28(2): 191.

COATES, A.G., COLLINS, L.S., AUBRY, M. \& BERGGREN, W.A., 2004: The Geology of 
the Darien, Panama, and the late MiocenePliocene collision of the Panama arc with northwestern South America.- Geol. Soc. Amer. Bull. 116: 1327-1344.

DALQUEST, W.W. \& MOOSER, O., 1980: Late Hemphillian mammals of the Ocote Local Fauna, Guanajuato, Mexico.- The PearceSellards Series, 32: 1-25.

GAUDIN, T. J. G., 2004: Phylogenetic relationships among sloths (Mammalia, Xenarthra, Tardigrada): the craniodental evidence.Zoological J. Linnean Soc. 140: 255-305.

HIRSCHFELD, S.E., 1981: Pliometanastes protistus (Edentata, Megalonychidae) from Knight's Ferry, California, with discussions of early Hemphillian megalonychids.- PaleoBios, 36: 1-16.

HIRSCHFELD, S.E. \& WEBB, S.D., 1968: PIioPleistocene megalonychid sloths of North America.- Bull. Florida State Mus. Biol. Sci. 12(5): 213-296.

HULBERT, R.C., 1988: Calippus and Protohippus (Mammalia, Perissodactyla, Equidae) from the Miocene (Barstovian-Early Hemphillian) of the Gulf Coastal Plain.Bull. Florida State Mus. Biol. Sci. 32(3): 221-340.

JOHNSTON, C. S. \& SAVAGE, D.E., 1955: A survey of various late Cenozoic vertebrate faunas of the panhandle of Texas. Part 1. Introduction, description of localities, preliminary faunal lists.- Univ. Calif. Publ. Bull. Dept. Geol. Sci. 31: 27-50.

LANCE, J.F., 1950: Paleontología y Estratigrafía del Plioceno de Yepómera, Estado de Chihuahua $1^{\text {a }}$ parte: Equidos, excepto Neohipparion.- Univ. Nac. Autónoma de México, Inst. de Geol. 54: 1-81.
LAURITO, C. \& VALERIO, A., 2005: First record of Rhynchotherium blicki (Frick, 1933) for the late Cenozoic of Costa Rica.Rev. Geol. Amér. Central, 33: 75-82.

LAURITO, C. \& VALERIO, A., 2008: The first record of Gavialosuchus americanus Sellards (1915) (Eusuchia: Crocodylidae, Tomistominae) for the Late Tertiary of Costa Rica and Central America.- Rev. Geol. Amér. Central, 39: 107-115.

LAURITO, C. \& VALERIO, A., 2010: Los caballos fósiles de la Formación Curré, cantón de Coto Brus, Costa Rica.- 131 págs. Mus. Nac. de Costa Rica, San José.

LAURITO, C.A. \& VALERIO, A.L., 2012: Paleobiogeografía del arribo de mamíferos suramericanos al sur de América Central de previo al gran intercambio biótico americano: Un vistazo al GABI en América Central.- Rev. Geol. Amér. Central, 46: 123-144.

LEITE, M.,1990: Stratigraphy and mammalian paleontology of the Ash Hollow Formation (Upper Miocene) on the north shore of Lake McConaughy, Keith County, Nebraska.Univ. of Wyoming, Contributions to Geol. 28: 1-29.

LINDSAY, E.H., 1984: Late Cenozoic Mammals from Northwestern Mexico.- En: MACFADDEN, B.J. (ed.): Origin and Evolution of the Cenozoic Vertebrate Fauna of Middle America.- J. Vert. Paleont. 4(2): 208-215.

LINDSAY, E.H., OPDYKE, N.D. \& JOHNSON, N.M., 1984: Blancan-Hemphillian Land Mammal Ages and Late Cenozoic Mammal Dispersal Events.- Annual Review of Earth and Planetary Sci. 1984: 445-488. 
MACFADDEN, B.J, 1977: Magnetic polarity stratigraphy of the Chamita Formation stratotype (Mio-Pliocene) of North-central New Mexico.- Amer. J. Sci. 277: 769-800

MACFADDEN, B.J., 1982: New species of primitive three-toed browsing horse from the Miocene phosphate mining district of central Florida. - Florida Scientist, 45: $117-125$.

MACFADDEN, B.J. \& DOBIE, J.L., 1998: Late Miocene Three-Toed Horse Protohippus (Mammalia, Equidae) from Southern Alabama.- J. Paleont. 72(1): 149-152.

MACFADDEN, B.J., FOSTER, D.A., RINCÓN, A.F., MORGAN, G.S., \& JARAMILLO, C., 2012: The New World Tropics as a cradle of biodiversity during the Early Miocene: calibration of the Centenario Fauna from Panama.- GSA Annual Meeting. North Carolina: 132.

MACPHEE, R.D.E., 2005: 'First' appearances in the Cenozoic land-mammal record of the Greater Antilles: significance and comparison with South American and Antarctic records.- J. Biogeography, 32: 551-564.

MACPHEE, R.D.E. \& ITURRALDE-VINENT, M.A., 1994: First Tertiary land mammal from Greater Antilles: an Early Miocene sloth (Xenarthra, Megalonychidae) from Cuba.- Amer. Mus. Novitates, 3094: 1-13.

MACPHEE, R.D.E. \& ITURRALDE-VINENT, M.A., 1995: Origin of the Greater Antillean land mammal fauna, 1: new Tertiary fossils from Cuba and Puerto Rico.- Amer. Mus. Novitates, 3141: 1-31.

MACPHEE, R.D.E. \& ITURRALDE-VINENT, M.A., 2012: When and how did land vertebrates reach the Greater Antilles?.- $72^{\text {nd }}$
Annual Meeting Society of Vertebrate Paleontology. North Carolina: 132.

MACPHEE, R.D.E., ITURRALDE-VINENT, M.A. \& GAFFNEY, E.S., 2003: Domo de Zaza, an Early Miocene vertebrate locality in south-central Cuba, with notes on the tectonic evolution of Puerto Rico and the Mona Passage.- Amer. Mus. Novitates, 3394: 1-42.

MAGUIRE, K.C., 2008: Paleobiogeography of Miocene to Pliocene Equinae of North America: A Phylogenetic Biogeographic and Niche Modeling Approach.- 195 págs. Ohio Univ., Ohio [Tesis M.Sc.].

MARSHALL, L.G., BUTLER, R.F., DRAKE, R.E., CURTIS, G.H. \& TEDFORD, R.H, 1979: Calibration of the Great American Interchange.- Science, 204: 272-279.

MAY, S.R. \& REPPENNING, C.A., 1982: New evidence for the age of the Mount Eden Fauna, Southern California.- J. Vert. Paleont. 2: 109-113.

MCDONALD, H.G., 2005: Palecology of extinct xenarthrans and The Great American Biotic Interchange.- Bull. Fla. Mus. Nat. Hist. 45(4): 319-340.

MCDONALD, H.G., 2005: Paleoecology of the extinct Xernarthras and The Great American Biotic Interchange.- En: HULBERT, R.C., MORGAN, G.S. \& BASKING, J.A. (eds.: Cenozoic Vertebrates of the Americas. Paper to Honor David S. Webb.- Bull. Fla. Mus. Nat. Hist. 45(4): 319-340.

MCDONALD, H.G. \& NAPLES, V.L., 2007: Chapter 10: Xenarthra.- En: JANIS, C.M., GUNNELL, G.F. \& UHEN, M.D. (eds): Evolution of Tertiary Mammals of North America, Vol. 2.- Cambridge University Press, Cambridge: 147-160. 
MORGAN, G.S., 2005: The Great American Biotic Interchange in Florida.- Bull. Florida Mus. Nat. Hist. 45(4): 271-311.

OLSON, E.C. \& MCGREW, P.O., 1941: Mammalian fauna from the Pliocene of Honduras.- Bull. Geol. Soc. Amer. 52: 1219-1244.

PASCUAL, R, 2006: Evolution and geography: The Biogeographic History of South American Land Mammals.- Ann. Missouri Bot. Gard. 93: 209-230.

PATTERSON, B. \& PASCUAL, R., 1968: Evolution of mammals on southern continents. The fossil mammal fauna of South America.- The Quarterly Rev. Biol. 43: 409-451.

SCHULTZ, G.E., 1990: Stop 15: Early Hemphillian faunas of the Texas and Oklahoma Panhandles.-En: GUSTAVSON, T.C. (ed.): Tertiary and Quaternary stratigraphy and vertebrate paleontology of parts of northwestern Texas and eastern New Mexico; Guidebook.- Univ. of Texas, Austin: 95-103.

SIMPSON, G.G., 1956: Zoogeography of West Indian Land Mammals.- Amer. Mus. Novitates, 1759: 1-28.

TEDFORD, R.H., 1981: Mammalian biochronology of the late Cenozoic Basins of New Mexico.- Geol. Soc. Amer. Bull. 92: 10081022.

TEDFORD, R.H., \& BARGHOORN, S.F., 1993: Neogene stratigraphy and mammalian biochronology of the Española Basin, northern New Mexico.- En: LUCAS, S.G. \& ZIDEK, J. (eds): Vertebrate paleontology in New Mexico.- New Mexico Mus. Nat. Hist. and Sci. Bull. 2: 159-168.
TEDFORD, R.H., GALUSHA, T., SKINNER, M.F., TAYLOR, B.E., FIELDS, R.W., MACDONALD, J.R., RENSBERGER, J.M., WEBB, S.D. \& WHISTLER, D.P., 1987: Faunal succession and biochronology of the Arikareean through Hemphillian interval (Late Oligocene through earliest Pliocene epochs) in North America.- En: WOODBURNE, M.O. (ed.): Cenozoic Mammals of North America, Geochronology and Biostratigraphy. - Univ of California Press, Berkeley: 153-210.

VALERIO, A.L., 2010: Paleontología, bioestratigrafía y paleoecología de los caballos fósiles de la Formación Curré en el cantón de Coto Brus, Costa Rica (análisis basado en material dental).- 353 págs. Univ. de Costa Rica, San José [Tesis Lic.].

VALERIO, A.L. \& LAURITO, C.A., 2008: Dental remains of inmature individuals of Rhynchotherium blicki (Frick, 1933) of San Gerardo de Limoncito, Coto Brus, Costa Rica.- Rev. Geol. Amér. Central, 39: 87-91.

WANG, X., TEDFORD, R.H. \& TAYLOR, B.E., 1999: Phylogenetic systematics of the Borophaginae (Carnivora: Canidae).- Bull. Amer. Mus. Nat. Hist. 243: 1-392.

WEBB, S.D., 1976: Mammalian faunal dynamics of the Great American Interchange.Paleobiol. 2: 216-234.

WEBB, S.D., 1985: Late Cenozoic mammal dispersals between the Americas. - En: STEHLI, F.G. \& WEBB, S.D. (eds): The Great American Biotic Interchange. Topics in Geobiology, (4): 357-386.

WEBB, S.D., 1989: Osteology and relationships of Thinobadistes segnis, the first mylodont sloth in North America. - En: REDFORD, 
K.H. \& EISENBERG, J.F. (eds): Advances in Neotropical Mammalogy. - The Sandhill Crane Press, 469-532.

WEBB, S.D., 2006: The Great American Biotic Interchange, Patterns and Processes.- Ann. Missouri Bot. Gard. 93: 245-257.
WHITE, J., \& MACPHEE, R.D.E., 2001: The sloths of the West Indies: a systematic and phylogenetic review.- En: WOODS, C.A. \& SERGILE, F.E. (eds): Biogeography of the West Indies: Patterns and Perspectives [2 $2^{\mathrm{a}}$ ed.].- CRC Press. Boca Ratón, Florida: 201-236. 